\title{
Thermomagnetic Study on Spinodal Decomposition of Ni-Cu-Pd Alloy
}

\author{
By Yoshinori Murata* and Yoshiro Iwama**
}

\begin{abstract}
A thermomagnetic approach was used to investigate the early stage of spinodal decomposition in the ferromagnetic $\mathrm{Ni}-29$ at $\% \mathrm{Cu}-21$ at $\% \mathrm{Pd}$ alloy. First, the phase relationship associated with the decomposition was determined by measuring the Curie temperature and the lattice parameter. Next it was confirmed by TEM observations that as annealed at $773 \mathrm{~K}$ after solution treatment, the alloy decomposes by a spinodal mechanism and result in the modulated structure. The thermomagnetic curves were measured for the alloy annealed at $773 \mathrm{~K}$ during various periods of time. By comparing the experimental curves with the calculated ones, assuming a sinusoidal composition fluctuation, the kinetics of the amplitude growth can be estimated in the spinodal decomposition.
\end{abstract}

(Received October 30, 1980)

\section{Introduction}

In all the $\mathrm{Ni}-\mathrm{Cu}, \mathrm{Ni}-\mathrm{Pd}$ or $\mathrm{Cu}-\mathrm{Pd}$ binary systems there is a continuous series of solid solutions, though only in the $\mathrm{Cu}-\mathrm{Pd}$ system there exists a tendency to form some ordered structures near the compositions of $\mathrm{Cu}_{3} \mathrm{Pd}$ and $\mathrm{CuPd}$ at lower temperatures ${ }^{(1)}$. In a ternary $\mathrm{Ni}-\mathrm{Cu}-\mathrm{Pd}$ system, however, there appears a miscibility gap in the solid state where two isomorphous phases $\gamma_{1}$ and $\gamma_{2}$ are in equilibrium with each other. According to Raub et $a l .{ }^{(2)}$ the break in solubility originates in the ternary system below about $950 \mathrm{~K}$ and extends over a region with composition less than 30 at $\% \mathrm{Pd}$. Thus, the miscibility gap space forms a dome shape in the spacial phase diagram. This is a typical ternary miscibility gap in a sense that it is independent of any reaction involved in the sub-binary systems. On appling the theory of spinodal decomposition to the ternary alloy, the spinodal surface, instead of the spinodal line in a usual binary alloy, will be formed inside the miscibility gap region, and it may also constitute a dome shape similar to the miscibility gap surface ${ }^{(3)}$. At an appropriate temperature below the spinodal surface, therefore, it must commence the

* Graduate School, Nagoya University, Furocho, Nagoya 464, Japan.

** Department of Applied Physics, Faculty of Engineering, Nagoya University, Furocho, Nagoya 464, Japan. decomposition in the spinodal mechanism. That is, in the early stage a compositional plane wave will be developed with a specified wavelength, resulting in a modulated structure composed of the two phases in the final equilibrium.

Now, the spinodal decomposition of the $\mathrm{Ni}-\mathrm{Cu}-\mathrm{Pd}$ alloy is of special interest, because one of the decomposition products is a ferromagnetic phase rich in $\mathrm{Ni}$, while the other is a paramagnetic phase rich in $\mathrm{Cu}$ and $\mathrm{Pd}$. In the early stage of decomposition, therefore, we may obtain an inhomogeneous ferromagnetic alloy with the composition fluctuation, especially with respect to $\mathrm{Ni}$ atoms. There are few thermomagnetic studies on such an inhomogeneous ferromagnetic alloy ${ }^{(4)}$, but the thermomagnetic curve obtained for the alloy would be expected to reveal an interesting aspect, reflecting the composition fluctuation involved.

In the present work an alloy with composition of $\mathrm{Ni}-29$ at $\% \mathrm{Cu}-21$ at $\% \mathrm{Pd}$ was chosen to investigate the process of the spinodal decomposition using the thermomagnetic approach. The purposes are, first, to carefully examine the phase relationship associated with the twophase separation reaction, and then, to verify that the decomposition is evidently of the spinodal type, and finally to carry out the thermomagnetic measurements on the specimens at various stages of decomposition. The results obtained will be discussed on the basis of the theory of spinodal decomposition. 


\section{Experimental Procedures}

The alloy specimens were prepared from Mond nickel (99.95\% purity), electrolytic copper (99.98\% purity) and metallic palladium (99.9\% purity). The materials were melted in Tammann furnace under an argon atmosphere and cast into an iron mould. The chemical compositions are shown in Table 1. After soaking at $1273 \mathrm{~K}$ for $86.4 \mathrm{ks}(24 \mathrm{~h})$, the ingots were machined into cylindrical specimens $5 \mathrm{~mm}$ long and $5 \mathrm{~mm}$ in dia. suitable for thermomagnetic measurement. All the specimens were solution annealed at $1273 \mathrm{~K}$ for $7.2 \mathrm{ks}(2 \mathrm{~h})$ followed by quenching into ice-brine to retain a single phase state. The specimens exhibited a normal shape of magnetization vs temperature curve, i.e., the $M-T$ curve, similar to that of a homogeneous ferromagnetic alloy. Their Curie temperatures were determined from an inflexion point on the respective $M-T$ curves.

Among these alloys one alloy was exclusively used to make a detailed thermomagnetic study of the process of spinodal decomposition, whose composition is $\mathrm{Ni}-28.8$ at $\% \mathrm{Cu}-21.0$ at $\% \mathrm{Pd}$ and corresponds to No. 6 alloy in Table 1, and it will be hereafter expressed as $\mathrm{Ni}-29$ at $\% \mathrm{Cu}-21$ at $\% \mathrm{Pd}$ using round numbers. Besides the rod specimens for thermomagnetic measurement, wire specimens $0.8 \mathrm{~mm}$ in dia. were prepared from the same ingot for an X-ray

Table 1 Chemical composition and Curie temperature of the specimens.

\begin{tabular}{|c|c|c|c|c|}
\hline \multirow{2}{*}{ Alloy No. } & \multicolumn{3}{|c|}{$\begin{array}{l}\text { Chemical composition } \\
(\text { at } \%)\end{array}$} & \multirow{2}{*}{$\begin{array}{c}\text { Curie } \\
\text { temperature }(K)\end{array}$} \\
\hline & $\mathrm{Ni}$ & $\mathrm{Cu}$ & $\mathbf{P d}$ & \\
\hline 1 & 49.7 & 40.6 & 9.7 & 182 \\
\hline 2 & 60.0 & 30.1 & 9.9 & 283 \\
\hline 3 & 70.0 & 19.9 & 10.1 & 395 \\
\hline 4 & 79.2 & 10.4 & 10.4 & 491 \\
\hline 5 & 40.5 & 38.9 & 20.6 & 160 \\
\hline 6 & 50.2 & 28.8 & 21.0 & 270 \\
\hline 7 & 60.2 & 19.0 & 20.8 & 371 \\
\hline 8 & 70.0 & 9.3 & 20.7 & 475 \\
\hline 9 & 30.4 & 37.6 & 32.0 & 118 \\
\hline 10 & 40.3 & 28.3 & 31.4 & 239 \\
\hline 11 & 51.0 & 15.7 & 33.3 & 334 \\
\hline 12 & 60.1 & 8.7 & 31.2 & 441 \\
\hline
\end{tabular}

diffraction experiment as well as a resistivity measurement at elevated temperatures. In addition, thin foil specimens were prepared from the ingot to perform transmission electron microscope (TEM) observation. The foil specimens were finished by electrolytic polishing in nitric acid methyl alcohol solution at $233 \mathrm{~K}$.

\section{Experimental Results}

\section{Determination of phase relationship}

Figure 1 shows the isothermal section at $773 \mathrm{~K}$ of the $\mathrm{Ni}-\mathrm{Cu}-\mathrm{Pd}$ ternary equilibrium diagram, which was proposed by Raub et al. ${ }^{(2)}$, on the basis of their measurement of the lattice parameter. The double circle in the figure denotes the composition of the present alloy. If it is quenched from a high temperature, say, $1273 \mathrm{~K}$, where the homogeneous solid solution is stable, and then annealed at $773 \mathrm{~K}$, it should decompose into two isomorphous phases, one is a Ni-rich $\gamma_{1}$ phase and the other a $\mathrm{Cu}, \mathrm{Pd}$ rich $\gamma_{2}$ phase, although the tie line, or conjugate line, has not yet determined accurately. Here, the chemical compositions of the phases in the final equilibrium at $773 \mathrm{~K}$ were estimated in the following way.

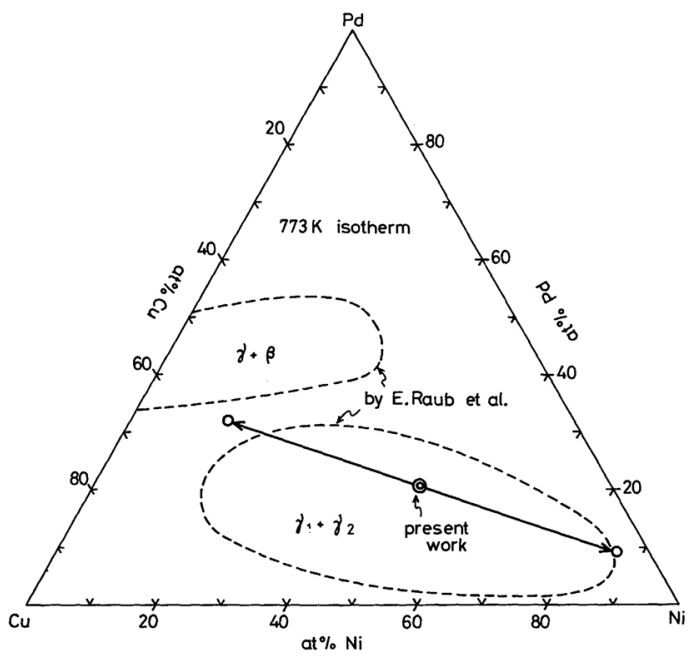

Fig. 1 The isothermal section at $773 \mathrm{~K}$ of the $\mathrm{Ni}-\mathrm{Cu}-$ Pd ternary system. Double circle is the alloy composition concerning with the present work and the straight line its conjugate line. Dashed line indicates two-phase regions by Raub et al. 
Figure 2 shows iso-lattice parameter lines for the homogeneous $\mathrm{Ni}-\mathrm{Cu}-\mathrm{Pd}$ alloys which are drawn according to the data of Raub et $a l .{ }^{(2)}$ As seen in the figure, they constitute a family of parallel lines with nearly equal spacing. On the other hand, the Curie temperature is also dependent on the composition, and the behavior is shown by the iso-Curie temperature lines in Fig. 3, which are drawn based on the present thermomagnetic measurements on the quenched specimens. On comparing both the figures, it can be clearly seen that the two

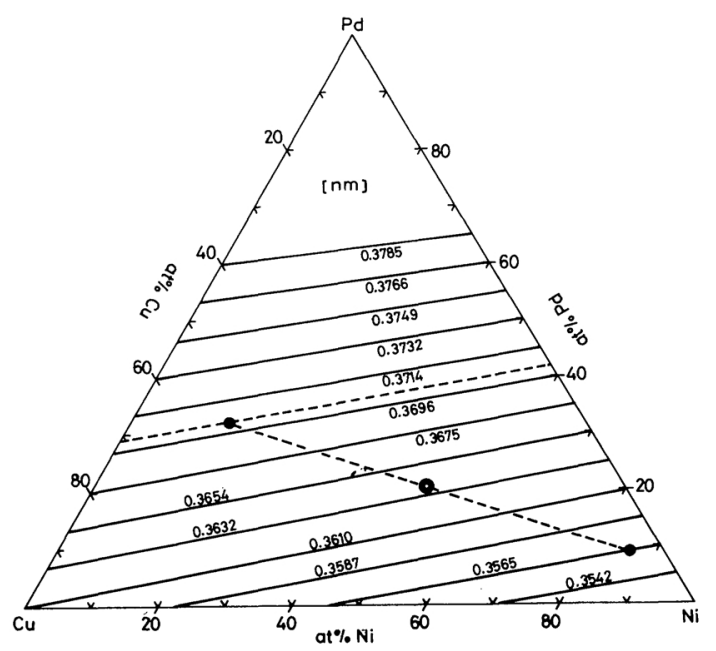

Fig. 2 The iso-lattice parameter lines in the Ni$\mathrm{Cu}-\mathrm{Pd}$ ternary system.

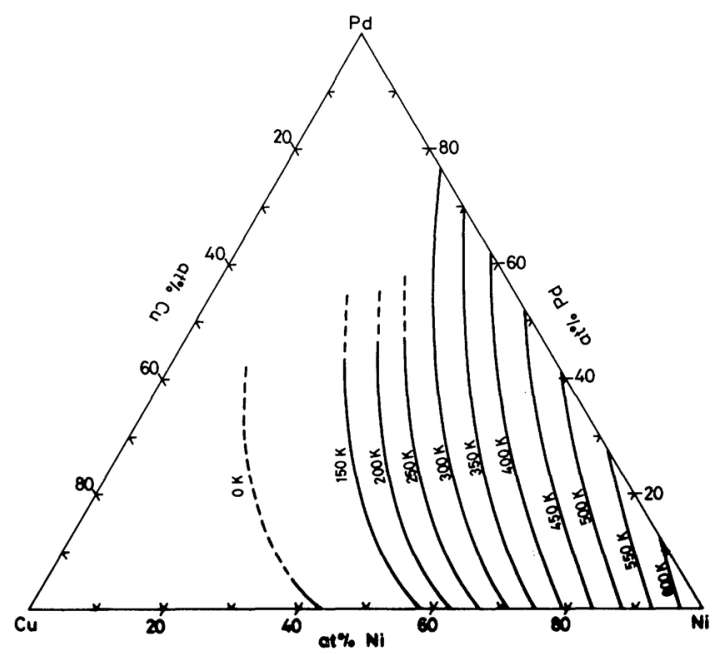

Fig. 3 The iso-Curie temperature lines in the Ni$\mathrm{Cu}-\mathrm{Pd}$ ternary system. sets of lines intersect each other at nearly right angles. This may imply that if a Curie temperature as well as a lattice parameter is measured for any specimen, the composition would be uniquely determined from the above data as a guide. Accordingly, the alloy with composition of $\mathrm{Ni}-29$ at $\% \mathrm{Cu}-21$ at $\% \mathrm{Pd}$ was annealed at $773 \mathrm{~K}$ for a week, which was long enough to ensure the complete decomposition into the two phases of $\gamma_{1}$ and $\gamma_{2}$. The $\mathrm{X}$-ray diffractometry revealed, then, that the lattice parameters of the $\gamma_{1}$ and $\gamma_{2}$ phases were $a_{1}=$ $0.3564 \mathrm{~nm}$ and $a_{2}=0.3703 \mathrm{~nm}$, respectively. Since only the $\gamma_{1}$ is ferromagnetic, the $M-T$ curve obtained for the alloy exhibits a single inflexion point at $560 \mathrm{~K}$, which can be taken as the Curie temperature of the $\gamma_{1}$ phase. Following the procedure described above, the values of lattice parameter and Curie temperature allowed to estimate the chemical composition of the $\gamma_{1}$ phase as $\mathrm{Ni}_{87} \mathrm{Cu}_{4} \mathrm{Pd}_{9}$. The other phase of $\gamma_{2}$ should lie on a tie line connecting the original composition (denoted by the double circle in Fig. 1) with that of the $\gamma_{1}$ phase. Consequently, a point at which the tie line intersects with the iso-lattice parameter line of $a=0.3703$ must give the composition of the $\gamma_{2}$ phase. The graphic approach is illustrated by a broken line in Fig. 2, and the resulting composition was determined as $\mathrm{Ni}_{15} \mathrm{Cu}_{53} \mathrm{Pd}_{32}$. Thus, it can be concluded that if the alloy with composition of $\mathrm{Ni}_{50} \mathrm{Cu}_{29} \mathrm{Pd}_{21}$ is quenched from a single phase region followed by annealing at $773 \mathrm{~K}$, it finally decomposes into the ferromagnetic $\gamma_{1}$ phase with composition of $\mathrm{Ni}_{87} \mathrm{Cu}_{4} \mathrm{Pd}_{9}$ and the paramagnetic $\gamma_{2}$ phase with composition of $\mathrm{Ni}_{15} \mathrm{Cu}_{53} \mathrm{Pd}_{32}$. The former Curie temperature is $560 \mathrm{~K}$, while the latter one is nearly $0 \mathrm{~K}$.

\section{Measurements of $\boldsymbol{M}-\boldsymbol{T}$ curves}

If the alloy is quenched from $1273 \mathrm{~K}$ into ice-brine, a homogeneous solid solution is formed, though exactly it should be called supersaturated. The thermomagnetic measurement will reveal a normal $M-T$ curve with a single inflexion point, unless any other phase transition occurs. This was indeed observed, as shown in Fig. 4. The Curie temperature was determined as $270 \mathrm{~K}$. Another approach of 


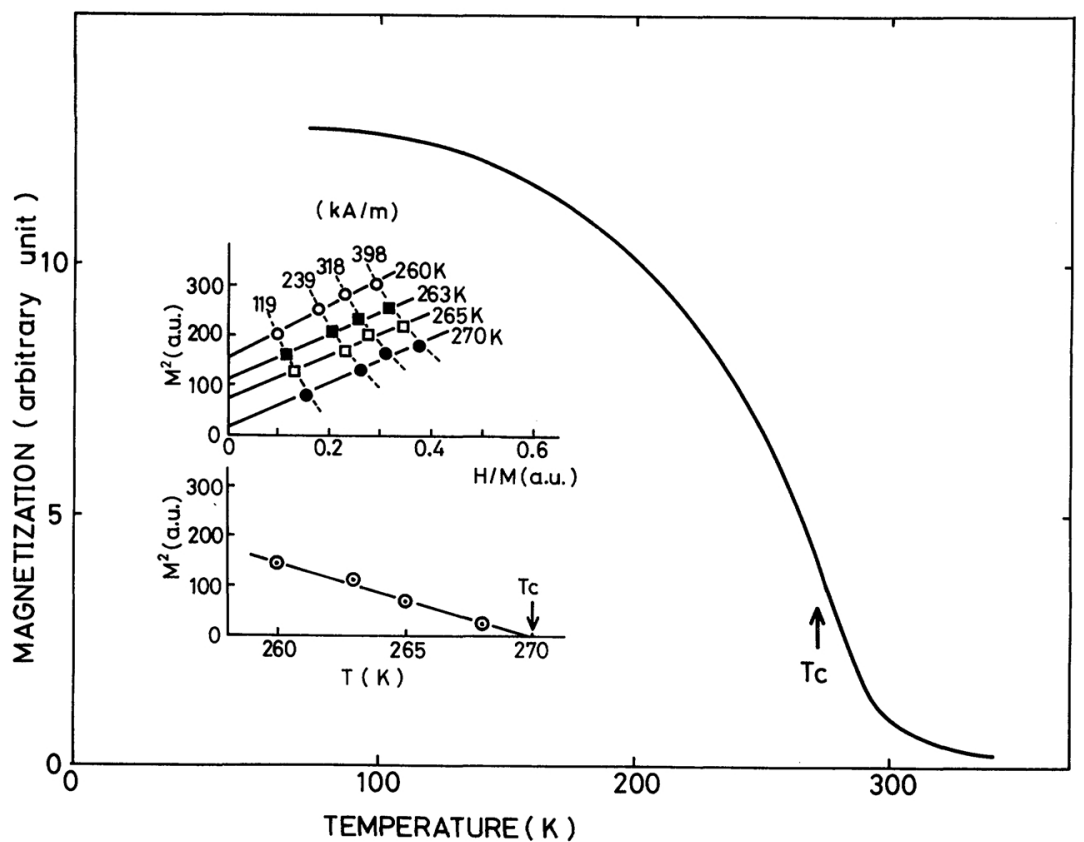

Fig. 4 Magnetization vs temperature curve of the quenched $50 \mathrm{Ni}-29 \mathrm{Cu}-21 \mathrm{Pd}$ alloy and the insets shows Arrott plotting of the same specimen.

Arrott plotting ${ }^{(5)}$ was also applied to the same specimen, but the result was found to give an identical value of $270 \mathrm{~K}$, as shown in the inset of Fig. 4.

According to the phase diagram concening the present alloy, the supersaturated alloy is expected to undergo the two-phase separation at $773 \mathrm{~K}$. If the reaction occurs in a mode of spinodal decomposition, at least in the early stage of annealing, composition fluctuation should be developed within the specimen. Thus, the thermomagnetic measurements were made on the specimens subjected to the annealing for various time durations. The $M-T$ curves

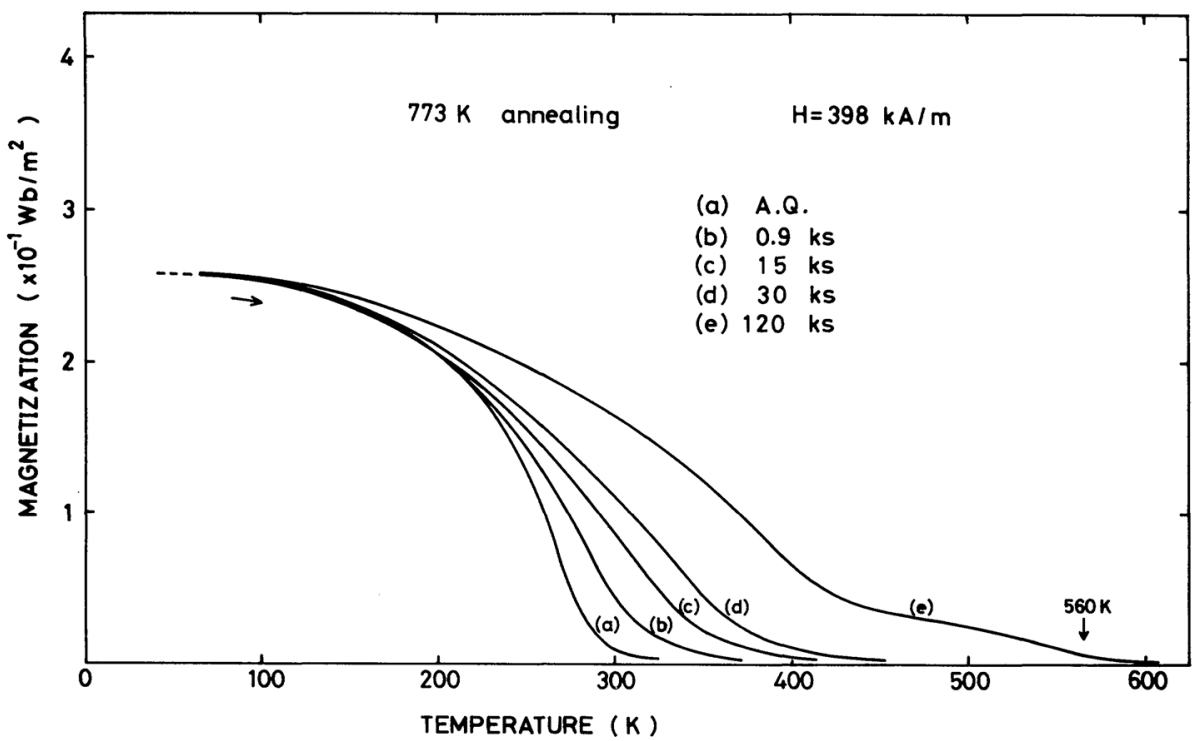

Fig. 5 Change of magnetization vs temperature curves with isothermal annealing at $773 \mathbf{K}$. 
obtained are shown in Fig. 5. Contrary to the result in Fig. 4, the $M-T$ curves exhibit an anomalous shape characterized by a long tail, and the tailing becomes more pronounced with increase in annealing time. The presence of the tail makes it difficult to determine a welldefined Curie temperature from the $M-T$ curves in terms of inflexion point. Of course, the Arrott plot also would not give an unambiguous Curie temperature. It is probable that such an inhomogeneous alloy does not have a unique but somewhat broad Curie temperature extended over a limited temperature range. As the annealing is further prolonged up to $120 \mathrm{ks}$ (ca. $2000 \mathrm{~min}$ ), the alloy seems to approach the final equilibrium at $773 \mathrm{~K}$. This is because Fig. 5 shows another inflexion point at $560 \mathrm{~K}$, which is close to the Curie temperature of the Ni-rich $\gamma_{1}$ phase in the final state.

\section{Resistivity measurements at elevated temperatures}

On the ferromagnetic metals and alloys, in general, it is well known that the resistivity vs temperature $(\rho-T)$ curve exhibits a distinct bend in the vicinity of the Curie temperature ${ }^{(6)}$. So, the $\rho-T$ curve was measured on the asquenched specimen as well as the one annealed at $773 \mathrm{~K}$ for $30 \mathrm{ks}(500 \mathrm{~min})$. In Fig. 6 are shown the results obtained. The results for the as-quenched specimen indicates a bend near $270 \mathrm{~K}$, which coincides with the Curie temperature determined from the $M-T$ curve of the same specimen. But the latter exhibits, instead of a sharp bend, a smooth curve extended from 200 to $500 \mathrm{~K}$ with a fairly large curvature. Hence, the $\rho-T$ curve seems to verify the above suggestion that the spinodally decomposed alloy may be characterized by the inhomogeneous structure with a broad spectrum of Curie temperature.

\section{Electron microscope observation}

The TEM observation was carried out on the foil specimens subjected to the same heat treatment as the above. The TEM micrograph is shown in Photo. 1, where a (100) plane is concerned. Obviously it reveals a modulated structure developed along the $\langle 100\rangle$ crystal-

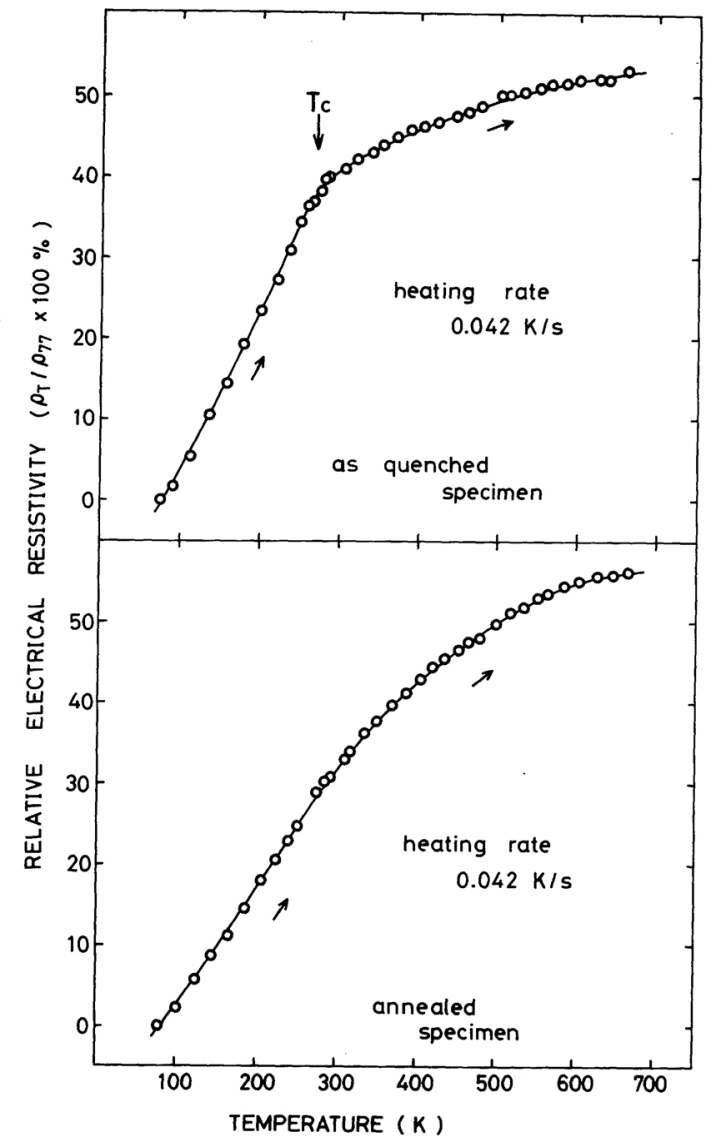

Fig. 6 Electrical resistivity vs temperature curves for the quenched and the annealed specimen.

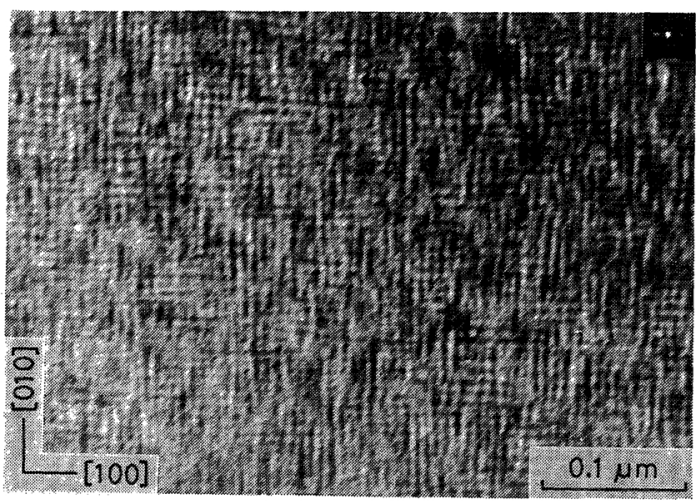

Photo. 1 Transmission electron micrograph of the $50 \mathrm{Ni}-29 \mathrm{Cu}-21 \mathrm{Pd}$ alloy annealed at $773 \mathrm{~K}$ for $30 \mathrm{ks}$. The inset shows 200 reflection spots with the satellites.

lographic directions, indicating that the precipitate structure must have resulted from the spinodal decomposition. A 200 diffraction 
spot shown in the inset of Photo. 1, is seen to accompany with some satellite spots, reflecting that the modulated structure is possibly derived from a certain periodic composition fluctuation $^{(7)}$. The wavelength could be evaluated as about $6.5 \mathrm{~nm}$ from the separation of the satellites.

\section{Discussion}

Among the experimental results so far described, it is the most striking that the spinodally decomposed alloy exhibits a peculiar shape of the $M-T$ curve characterized by a long tail, as shown by the curves (c) and (d) in Fig. 5. We are now in a position to interpret the results in terms of the theory of spinodal decomposition.

According to the theory of spinodal decomposition $^{(8)(9)}$, a sinusoidal form of composition fluctuation will be developed in the early stage of annealing. That is, the composition $c(r, t)$ at the position $r$ in the specimen and the time $t$ is represented by

$$
c(\boldsymbol{r}, t)-c_{0}=\int A(\boldsymbol{\beta}, t) \exp (i \boldsymbol{\beta}, \boldsymbol{r}) \mathrm{d} \boldsymbol{\beta},
$$

where $c_{0}$ is the initial composition of the alloy and $A(\beta, t)$ the amplitude of the Fourier component of the fluctuation at time $t$ with wavenumber vector $\boldsymbol{\beta}(=2 \pi / \lambda$ where $\lambda$ is the wavelength). For the early stages of spinodal decomposition a component with a certain wavelength $\lambda_{\mathrm{m}}$ has so sharp maximum growth rate that even in a very short period the compositional wave with $\lambda_{\mathrm{m}}$ may dominate over any other components. In the present case it has been proved to be $\lambda_{\mathrm{m}}=6.5 \mathrm{~nm}$.

Furthermore, in a cubic crystalline solid the decomposition waves are predicted to grow selectively along the $\langle 100\rangle$ directions due to the anisotropy of the elastic properties ${ }^{(10)}$. As a result the composition variation can be written as

$$
\begin{aligned}
c-c_{0}= & \Delta c[\sin (2 \pi x / \lambda)+\sin (2 \pi y / \lambda) \\
& +\sin (2 \pi z / \lambda)],
\end{aligned}
$$

where $\Delta c$ is the amplitude of the composition wave as restricted to a one-dimensional wave, and $x, y$ and $z$ mean three cubic axes. This equation defines a three-dimensional sinusoidal variation of composition along the three principal axes. It follows that within the specimen the composition variation, i.e., $c-c_{0}$ must fluctuate between the maximum value of $3 \Delta c$ and the minimum of $-3 \Delta c$. Further, it should be noted that the variation limits are to be confined on the tie line indicated in Fig. 2.

This composition fluctuation was divided into 24 equal sub-intervals of composition, and the volume fraction of each composition division was numerically calculated, yielding the hystogram shown in Fig. 7. The profile of the hystogram should be independent either of $\Delta c$ or $\lambda$, though the magnitude of the sub-interval is directly proportional to $\Delta c$. Thus, the inhomogeneous alloy can be assumed as an assembly of small regions of homogeneous alloys with various compositions. If further assumed that there exists no interaction between the small regions, the $M-T$ curve of the inhomogeneous alloy can be calculated as the superposition of the $M-T$ curves for the homogeneous regions with respective compositions. This approach is quite similar to that used in the

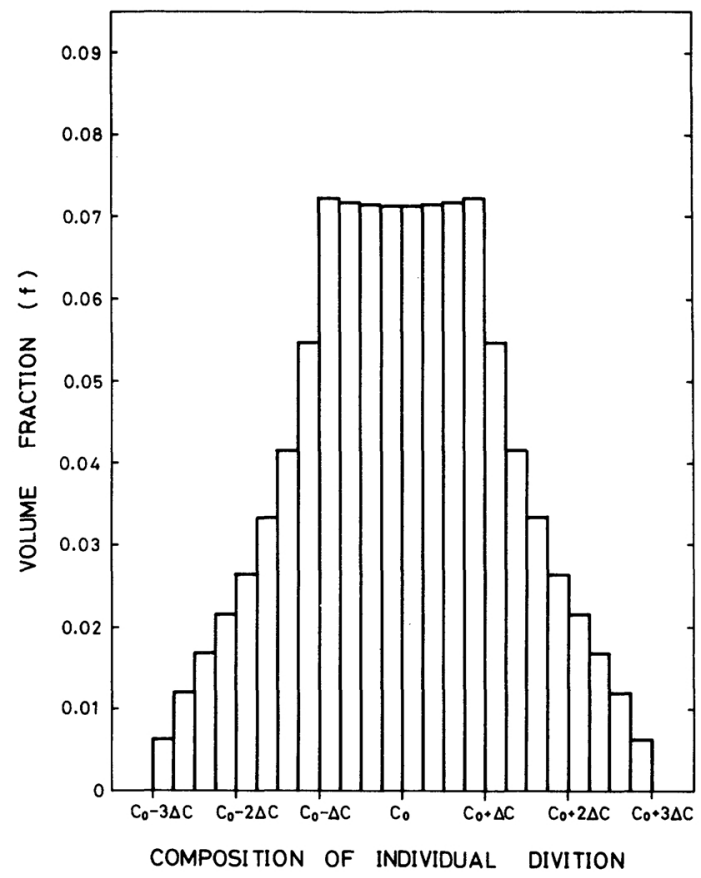

Fig. 7 Histogram showing the volume fraction of the respective composition divisions. 
Mössbauer study of the spinodal decomposition in $\mathrm{Fe}-\mathrm{Cr}-\mathrm{Co}$ alloys by Tahara et al..$^{(1)}$

For simplicity of calculation, we will hereafter, instead of the ternary composition $c$, the nickel content $C$ to specify the alloy composition. Referring back to Fig. 1, then, the permissible range of the composition is limited from $C_{\min }=0.15$ to $C_{\max }=0.86$ around the middle point of $C_{0}=0.50$. Over the whole range, composition dependencies of Curie temperature $\left(T_{\mathrm{c}}\right)$ and magnetic moment at $0 \mathrm{~K}\left(M_{0}\right)$ are shown in Fig. 8. While the former (indicated by open circles) was read off from the iso-Curie temperature lines in Fig. 3, the latter (indicated by closed circles) was determined from the measurements on several alloys freshly prepared.

If both values of $M_{0}$ and $T_{\mathrm{c}}$ are given for a homogeneous alloy, the $M-T$ curve can be easily calculated according to the molecular field approximation ${ }^{(12)}$. The $M-T$ relationship is, then, expressed as

$$
M / M_{0}=\tanh \left[\left(M / M_{0}\right) /\left(T / T_{\mathrm{c}}\right)\right],
$$

where $M$ means a magnetic moment at temperature $T$. The transcendental equation can be numerically solved using a computer prepared with a suitable sub-routine program, yielding the $M-T$ curve. In the case of the inhomogeneous alloy also, the similar calculation may be performed for the small homogeneous regions, subdivided in the manner previously described. If the weighted average of the $M-T$ curves thus obtained, is calculated by taking account of the hystogram in Fig. 7, we can obtain the $M-T$ curve of the inhomogeneous alloy in which the sinusoidal composition fluctuation is developed with a certain amplitude.

The curves calculated for the various am-

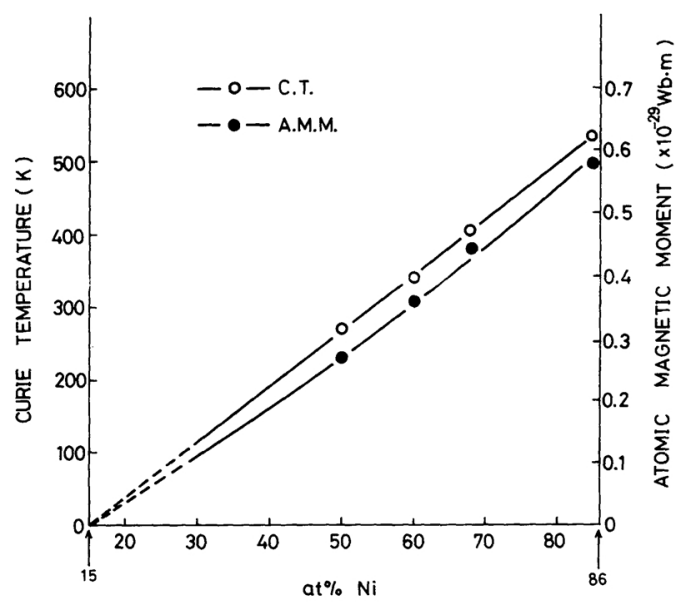

Fig. $8 \mathrm{Ni}$ content dependences of Curie temperature (open circle) and atomic magnetic moment (closed circle).

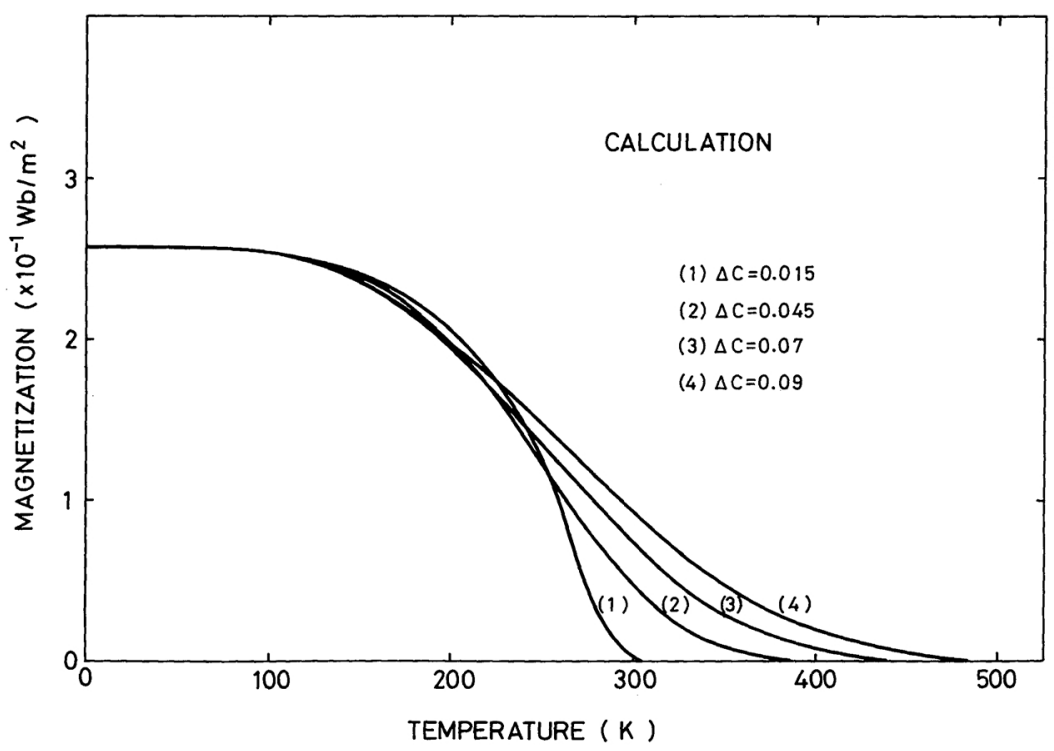

Fig. 9 Calculated curves of magnetization vs temperature with various amplitude of the sinusoidal composition fluctuation. 
plitudes are shown in Fig. 9, where the amplitude is represented by three-dimensional expression $3 \Delta C$ in terms of nickel content, which corresponds to the real amplitude of sinusoidal composition wave in the alloy. Being normalized by the maximum permissible amplitude $(=0.355)$, the values of $3 \Delta C=0.045$, $0.135,0.21$ and 0.27 are reduced to $0.13,0.38$, 0.59 and 0.76 , respectively. Comparing the calculated curves with the experimental ones shown in Fig. 5, a considerable resemblance is found in the overall aspect. First of all, the magnetic moment at temperatures lower than $100 \mathrm{~K}$ is kept unchanged independently of either the amplitude or the annealing time. With increasing amplitude from 0.135 to 0.27 , the tail in the calculated curve grows toward higher temperatures, consistent with the experimental curves, in which the tail grows as the increase in the annealing time of $0.9,15$ and $30 \mathrm{ks}(15,250,500 \mathrm{~min})$, respectively. The reasonable agreement clearly indicate that, during the annealing, the supersaturated solid solution does spinodally decompose in such a way that the sinusoidal composition fluctuation grows with a gradual increase in amplitude. Furthermore, by collating the experimental curves with the calculated ones, it is possible to semi-quantitatively, at least, estimate the amplitude of the composition wave. It is natural, however, that an exact fitting should never been expected between the experimental and calculated curves, because we have used a rough approximation of molecular field to calculate the $M-T$ curves.

In the present case, thus it could be reasonably recognized that, as the annealing time was increased $0.9,15$ and $30 \mathrm{ks}$, the amplitude of composition wave was allowed grow successively 38,59 and $76 \%$ of the final composition variation. Particularly, it is noticeable that even after the prolonged annealing of 30 ks (500 $\mathrm{min})$ at $773 \mathrm{~K}$, a sinusoidal form of composition fluctuation still remains, indicating that the final two-phase state has not yet been attained. Thus, it appears that the reaction rate associated the spinodal decomposition is rather slow.

In conclusion it should be emphasized that the thermomagnetic study combined with a suitable theoretical analysis is capable of elucidating the growth behavior of amplitude in the modulated structure derived from the spinodal decomposition in such a ferromagnetic $\mathrm{Ni}-\mathrm{Cu}-$ $\mathrm{Pd}$ alloy. While X-ray or electron microscope study can reveal the wavelength of the modulated structure, the present approach certainly provides information about the amplitude, complementary to the former. It may be worthy of special mention, because there are a few works on the amplitude analysis among many of experimental studies on the spinodal decomposition of alloys.

\section{REFERENCES}

(1) M. Hansen: Constitution of Binary Alloys, McGraw-Hill, New York, (1958), p. 612.

(2) E. Raub, O. Loebich, Jr., W. Plate and H. Krill: Z. Metallk., 62 (1971), 826.

(3) J. E. Meijering: Philips Res. Rept., 5 (1950), 333; 6 (1951), 183.

(4) D. H. Ben Isreal and M. E. Fine: Acta Met., 11 (1963), 1051.

(5) T. R. Mcguire and P. J. Flanders: Magnetism and Metallurgy Vol. I, A. E. Berkowitz and E. Kneller ed., Academic Press, (1969), p. 132.

(6) D. H. Martin: Magnetism in Solids, London Iliffe Books Ltd., (1967), p. 28, p. 74.

(7) V. Daniel and H. Lipson: Proc. R. Soc., A181 (1943), 368.

(8) J. W. Cahn: Acta Met., 9 (1961), 795.

(9) J. W. Cahn: Trans. Met. Soc. AIME, 242 (1968), 166.

(10) J. W. Cahn: Acta Met., 10 (1962), 179.

(11) R. Tahara, Y. Nakamura, M. Inagaki and Y. Iwama: Phys Status Solidi. (a), 41 (1977), 451.

(12) R. M. Bozorth: Ferromagnetism, D. Van Nostrand Company, Ltd., New York, (1951), 716. 